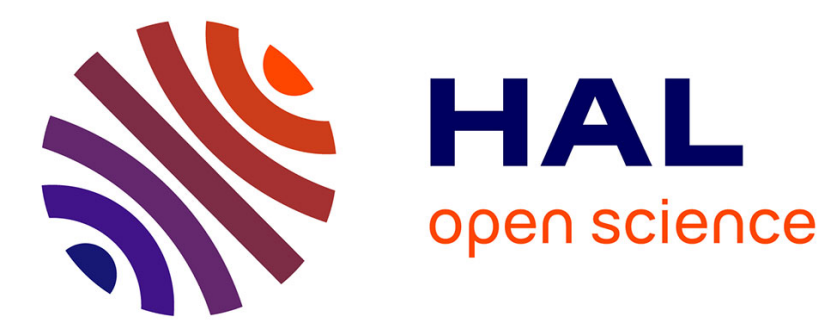

\title{
Coordination in networks of linear impulsive agents
}

\author{
Irinel-Constantin Morarescu, Samuel Martin, Antoine Girard
}

\section{To cite this version:}

Irinel-Constantin Morarescu, Samuel Martin, Antoine Girard. Coordination in networks of linear impulsive agents. 53rd IEEE Conference on Decision and Control, Dec 2014, Los Angeles, United States. pp.3713-3718, 10.1109/CDC.2014.7039967 . hal-01076175

\section{HAL Id: hal-01076175 \\ https://hal.science/hal-01076175}

Submitted on 21 Oct 2014

HAL is a multi-disciplinary open access archive for the deposit and dissemination of scientific research documents, whether they are published or not. The documents may come from teaching and research institutions in France or abroad, or from public or private research centers.
L'archive ouverte pluridisciplinaire HAL, est destinée au dépôt et à la diffusion de documents scientifiques de niveau recherche, publiés ou non, émanant des établissements d'enseignement et de recherche français ou étrangers, des laboratoires publics ou privés. 


\title{
Coordination in networks of linear impulsive agents ${ }^{1}$
}

\author{
Irinel-Constantin Morărescu, Samuel Martin, Antoine Girard ${ }^{2}$
}

October 21, 2014

${ }^{1}$ This work was funded by the ANR project COMPACS - "Computation Aware Control Systems", ANR-13-BS03-004.

${ }^{2}$ I.-C. Morărescu and S. Martin are with Université de Lorraine, CRAN, UMR 7039 and CNRS, CRAN, UMR 7039, 2 Avenue de la Forêt de Haye, Vandœuvre-lès-Nancy, France. A Girard is with Laboratoire Jean Kuntzmann, Université de Grenoble, B.P. 53, 38041 Grenoble, France. e-mails: constantin.morarescu@univ-lorraine.fr, samuel.martin@univ-lorraine.fr, antoine.girardeimag.fr 


\begin{abstract}
The paper focuses on consensus in heterogeneous networks containing both linear and linear impulsive dynamics. This model applies for networks that are formed by several clusters. Most agents can only update their state in a continuous way using innercluster agent states. On top of this, few agents also have the peculiarity to update their states in a discrete way by reseting it using states from agents outside their clusters. The motivation of this behavior is that communication constraints hamper continuous inter-clusters interactions. Under appropriate assumptions we prove that all subsystems asymptotically agree and we provide an upper-bound of the convergence speed. We illustrate the behavior with an academic example containing five agents grouped in two clusters.
\end{abstract}




\subsection{Introduction}

The problem of consensus or synchronization is motivated by different applications as communication networks, power and transport grids, decentralized computing networks, and social networks. Many of the existing works model the network as a graph with nodes and edges representing the systems and their interconnections, respectively. The connectivity of the network, persistence of links and interactions reciprocity influence the convergence speed $[1,2]$ and the achievement of consensus wether the dynamics is linear $[3,4,5,6,7]$, or nonlinear $[8,9,10]$. For this reason, most of the studies assume connectivity over bounded or unbounded time intervals. However, there also exist analysis and control designs for network connectivity preservation $[11,12,13]$ as well as studies of networks that loose connectivity property $[14,15]$.

Our point of view is that real networks are formed by several clusters inside which the interactions take place often and can be seen as continuous while, due to communication constraints (harsh environment, energy optimization or opinion preferences for instance), the inter-clusters interactions are rare, thus discrete. This leads us to a network dynamics that is expressed in term of reset systems (see $[16,17,18]$ for details). In [19] the authors assumed that each cluster has a leader and all the leaders nearly-periodically reset their state by taking into account the state of their neighboring leaders. However, generally we can have several agents in the same cluster that interact in discrete manner with agents in other clusters and, more importantly, we cannot synchronize the inter-clusters interactions in a decentralized way. Therefore, in this paper we address the more general and realistic problem of decentralized synchronization in heterogeneous networks containing both linear and linear impulsive dynamics

The rest of the paper is organized as follows. In Section 0.2 we introduce the concepts necessary for the problem formulation. Section 0.3 contains the work assumptions on the network structure and system dynamics. We also provide there, a prerequisite property of the exponential of a Laplacian matrix. The main results concerning the convergence analysis are presented in Section 0.4. Before conclusions we illustrate numerically the behavior of the network under consideration.

Notation. The following standard notation will be used throughout the paper. The sets of nonnegative integers, real and nonnegative real numbers are denoted by $\mathbb{N}, \mathbb{R}$ and $\mathbb{R}_{+}$, respectively. For a vector $x$ we denote by $\|x\|$ its Euclidian norm. The transpose of a matrix $A$ is denoted by $A^{\top}$. Given the matrices $A, B \in \mathbb{R}^{n \times n}$, notation $A \geq B(A>B)$ means that $A-B$ has all its entries (strictly) positive. By $I_{k}$ we denote the $k \times k$ identity matrix. $\mathbf{1}_{k}$ and $\mathbf{0}_{k}$ are the column vectors of size $k$ having all the components equal 1 and 0 , respectively. We also use $x\left(t_{k}^{-}\right)=\lim _{t \rightarrow t_{k}, t<t_{k}} x(t)$.

\subsection{Problem formulation}

We consider a network of $n$ agents described by the digraph (i.e. directed graph) $\mathcal{G}=$ $(\mathcal{V}, \mathcal{E})$ where the vertex set $\mathcal{V}$ represents the set of agents and the edge set $\mathcal{E} \subset \mathcal{V} \times \mathcal{V}$ represents the interactions.

Definition $1 A$ directed path of length $p$ in a given digraph $\mathcal{G}=(\mathcal{V}, \mathcal{E})$ is a union of directed edges $\bigcup_{k=1}^{p}\left(i_{k}, j_{k}\right)$ such that $i_{k+1}=j_{k}, \forall k \in\{1, \ldots, p-1\}$.

The node $j$ is connected with node $i$ in a digraph $\mathcal{G}=(\mathcal{V}, \mathcal{E})$ if there exists at least a directed path in $\mathcal{G}$ from $i$ to $j$ (i.e. $i_{1}=i$ and $j_{p}=j$ ).

A strongly connected digraph is such that any two distinct nodes are connected. A 
strongly connected component of a digraph is a maximal subset such that any of its two distinct nodes are connected.

We say node $i$ is a parent of node $j$ in the digraph $\mathcal{G}=(\mathcal{V}, \mathcal{E})$ if $(i, j) \in \mathcal{E}$. A directed tree is a directed subgraph in which there exists a single node without parents called root while all the others have exactly one parent. The length of a directed tree is the length of its longest path. A directed spanning tree of a digraph is a directed tree that contains all the nodes of the graph.

In the sequel, we consider that the vertex set $\mathcal{V}$ is partitioned in $m$ clusters $\mathcal{C}_{1}, \ldots, \mathcal{C}_{m}$ such that $\mathcal{C}_{i} \cap \mathcal{C}_{j}=\emptyset, \forall i \neq j$ and $\bigcup_{i=1}^{m} \mathcal{C}_{i}=\mathcal{V}$. Let us also introduce the graph $\mathcal{G}^{\prime}=\left(\mathcal{V}, \mathcal{E}^{\prime}\right)$ containing only the edges of $\mathcal{G}$ that connect agents belonging to the same cluster. That is

$$
\mathcal{E}^{\prime}=\left\{(i, j) \in \mathcal{E} \mid \exists k \in\{1, \ldots, m\} \text { such that } i, j \in \mathcal{C}_{k}\right\} .
$$

The state of each agent evolves continuously by taking into account the states of other agents belonging to the same cluster. Doing so, the agents approach local agreements which can be different from one cluster to another. In order to reach the consensus in the entire network every inter-clusters connection is activated at some discrete instants. When the inter-clusters link $(j, i) \in \mathcal{E} \backslash \mathcal{E}^{\prime}$ is activated, the state of agent $i$ is reset to a weighted average of the states of $i$ and $j$. If several links arriving at $i$ are activated simultaneously, all the source states of these edges are considered in the weighted average. For the sake of simplicity we define the clusters as follows:

$$
\mathcal{C}_{i}=\left\{m_{i-1}+1, \ldots, m_{i}\right\}, \forall i \in\{1, \ldots, m\}
$$

where $m_{0}=0, m_{m}=n$ and the cardinality of $\mathcal{C}_{i}$ is given by

$$
\left|\mathcal{C}_{i}\right| \triangleq n_{i}=m_{i}-m_{i-1}, \forall i \geq 1
$$

In order to keep the presentation simple each agent will have a scalar state denoted by $x_{i}$. We also introduce the vectors $x=\left(x_{1}, \ldots, x_{n}\right)^{\top} \in \mathbb{R}^{n}$ collecting the states of all the agents and $x_{\mathcal{C}_{i}}=\left(x_{m_{i-1}+1}, \ldots, x_{m_{i}}\right)^{\top} \in \mathbb{R}^{n_{i}}, i \in\{1, \ldots, m\}$ collecting the states of the agents belonging to cluster $i$, respectively.

We are ready now to introduce the linear reset system describing the overall network dynamics:

$$
\begin{cases}\dot{x}(t)=-L x(t), & \forall t \in \mathbb{R}_{+} \backslash \mathcal{T} \\ x\left(t_{k}\right)=P\left(t_{k}\right) x\left(t_{k}^{-}\right) & \forall k \in \mathbb{N} \\ x(0)=x_{0} & \end{cases}
$$

where $x_{0} \in \mathbb{R}^{n},\left(t_{k}\right)_{k}$ is the countable, diverging, increasing sequence of reset instants denoted by $\mathcal{T}, L \in \mathbb{R}^{n \times n}$ is a generalized Laplacian matrix associated to the graph $\mathcal{G}^{\prime}$ and $P\left(t_{k}\right) \in \mathbb{R}^{n \times n}$ is a stochastic matrix associated to the graph $\mathcal{G}\left(t_{k}\right)=\left(\mathcal{L}, \mathcal{E}\left(t_{k}\right)\right)$ where $\mathcal{E}\left(t_{k}\right) \neq \emptyset$ is the set of inter-clusters links activated at time $t_{k}$. We highlight that two consecutive reset instants can be arbitrarily close. The entries of $L$ and $P\left(t_{k}\right)$ satisfy the following relations:

$$
\left\{\begin{array}{l}
L_{i, j}=0, \text { if }(j, i) \notin \mathcal{E}^{\prime} \\
L_{i, j}<0, \text { if }(j, i) \in \mathcal{E}^{\prime}, i \neq j \\
L_{i, i}=-\sum_{j \neq i} L_{i, j}, \forall i \in\{1, \ldots, n\}
\end{array},\right.
$$




$$
\left\{\begin{array}{l}
P_{i, j}\left(t_{k}\right)=0, \text { if }(j, i) \notin \mathcal{E}\left(t_{k}\right) \\
P_{i, i}\left(t_{k}\right)>0, \forall i \in\{1, \ldots, n\} \\
P_{i, j}\left(t_{k}\right)>0, \text { if }(j, i) \in \mathcal{E}\left(t_{k}\right), i \neq j \\
\sum_{j=1}^{n} P_{i, j}\left(t_{k}\right)=1, \forall i \in\{1, \ldots, n\}
\end{array} .\right.
$$

According to (4), if $P_{i, j}\left(t_{k}\right)=0, \forall j \neq i$ then $P_{i, i}=1$, meaning that no jump occurs on the state of the agent $i$ at time $t_{k}$. The values $L_{i, j}$ and $P_{i, j}\left(t_{k}\right)$ represent the weight of the state of the agent $j$ in the updating process of the state of agent $i$ when using the continuous and discrete dynamics, respectively. The matrices $L$ and $P\left(t_{k}\right)$ describe the level of influence of each agent inside its cluster and outside it, respectively. It is worth noting that $L$ has the following block diagonal structure

$$
L=\left(\begin{array}{ccc}
L_{1} & & \\
& \ddots & \\
& & L_{m}
\end{array}\right), L_{i} \in \mathbb{R}^{n_{i}}
$$

with $L_{i} \mathbf{1}_{n_{i}}=\mathbf{0}_{n_{i}}$ and $P\left(t_{k}\right) \mathbf{1}_{m}=\mathbf{1}_{m}$.

\subsection{Preliminaries}

\subsubsection{Framework assumptions}

In order to prove that the reset algorithm (2) guarantees asymptotic consensus for every initial condition $x_{0}$ we have to impose some standard assumptions. The first one concerns a minimal connectivity property of the whole network and of each cluster.

Assumption 1 (Network structure) The graph $\mathcal{G}=(\mathcal{V}, \mathcal{E})$ is such that

- each cluster $\mathcal{C}_{i}$ contains a spanning tree formed with links belonging to $\mathcal{E}^{\prime}$,

- eventually re-ordering the clusters, the following holds: for all $i \geq 2$ there exist $j<i, l_{j} \in \mathcal{C}_{j}$ and $r_{i}$ a root of $\mathcal{C}_{i}$ such that $\left(l_{j}, r_{i}\right) \in \mathcal{E}$.

The previous assumption is satisfied if $\mathcal{G}$ is strongly connected. It also holds if there exists a tree between roots of all the clusters. We note that Assumption 1 implies 0 is simple eigenvalue of each $L_{i}, \forall i \in\{1, \ldots, m\}$ (see [4]).

Next hypothesis of this work is standard in the literature (see [20]) and it ensures a minimal influence of the states implicated in the reset process of the agents.

Assumption 2 (Minimal influence) There exists a positive constant $\alpha$ such that, for all reset times $t_{k}$, if $P_{i, j}\left(t_{k}\right) \neq 0, i, j \in\{1, \ldots, n\}$ then $P_{i, j}\left(t_{k}\right) \geq \alpha$.

In order to emphasize that agents belonging to the cluster $\mathcal{C}_{i}$ reset their state at time $t_{k} \in \mathcal{T}$ we use extraction function $\phi_{i}$ defined such that $t_{\phi_{i}(h)}, h \in \mathbb{N}$ is the $h$-th time an agent in cluster $\mathcal{C}_{i}$ resets its state, i.e.

$$
\phi_{i}(h+1)=\min \left\{k>\phi_{i}(h) \mid \exists u \in \mathcal{C}_{i}, v \in \mathcal{V} \backslash \mathcal{C}_{i}, P_{u, v}\left(t_{k}\right)>0\right\} .
$$

where $t_{\phi_{i}(0)}=0, \forall i \in\{1, \ldots, m\}$. We do not disregard the situation when agents from different clusters reset their state simultaneously. Therefore, we may have $\phi_{i}(k)=$ $\phi_{j}(h)$ for $i \neq j$ and $k, h \in \mathbb{N}$.

As we shall see in the sequel, Assumption 2 is analogous to a dwell time condition for the continuous dynamics. 
Assumption 3 (Dwell time) There exists a positive constant $\delta$ such that

$$
t_{\phi_{i}(k+1)}-t_{\phi_{i}(k)} \geq \delta, \forall i \in\{1, \ldots, m\} .
$$

In other words, there exists a lower bound for the period between the consecutive reset instants on the state of agents belonging to the same cluster. This bound guarantees that, between two consecutive reset instants of one cluster, the maximal distance between the states of the cluster is reduced.

Remark 1 A simple manner to ensure Assumption 3 in a decentralized way is the following. We suppose that each cluster possesses only one agent with the ability to interact outside its cluster and this agent activates its inter-clusters interactions respecting the dwell time condition.

It is important to mention that dynamics (2) leads to the collective state trajectory

$$
\left\{\begin{array}{l}
x(t)=e^{-L\left(t-t_{k}\right)} P\left(t_{k}\right) x\left(t_{k}^{-}\right), \quad \forall k \in \mathbb{N}, \forall t \in\left[t_{k}, t_{k+1}\right) \\
x(0)=x_{0}
\end{array}\right.
$$

but a jump occurs in $x_{\mathcal{C}_{i}}$ only at the time $t_{\phi_{i}(k)}$ involving edges with the sink in cluster $i$. This can be formalized as

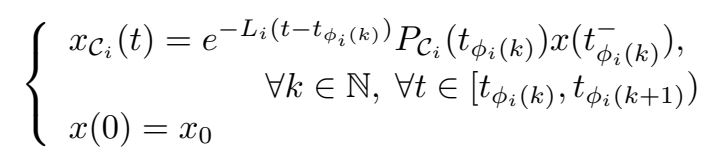

where $P_{\mathcal{C}_{i}}\left(t_{\phi_{i}(k)}\right)$ contains only the rows of $P\left(t_{\phi_{i}(k)}\right)$ corresponding to the cluster $\mathcal{C}_{i}$ (i.e. the rows $m_{i-1}+1, \ldots, m_{i}$ of $\left.P\left(t_{\phi_{i}(k)}\right)\right)$.

Next assumption ensures the presence of resets frequently enough and, as will be shown, force the local agreements of different clusters to converge one toward another.

Assumption 4 There exists a positive constant $\delta_{\max }>\delta$ satisfying the following:

- $\forall(i, j) \in \mathcal{E} \backslash \mathcal{E}^{\prime}$ there exists $t_{k} \leq \delta_{\text {max }}$ such that $(i, j) \in \mathcal{E}\left(t_{k}\right)$

- if $(i, j) \in \mathcal{E}\left(t_{k}\right)$ there exists $T \in\left[t_{k}+\delta, t_{k}+\delta_{\text {max }}\right]$ such that $(i, j) \in \mathcal{E}(T)$.

Remark 2 Notice that Assumption 4 implies that for all cluster $i$ and for all $k \in \mathbb{N}$,

$$
t_{\phi_{i}(k+1)}-t_{\phi_{i}(k)} \leq \delta_{\max } .
$$

Assumption 4 can be easily imposed in a decentralized way since it concerns the interclusters links one by one in a decoupled manner.

\subsubsection{Matrix prerequisite properties}

In this subsection we provide an instrumental result concerning the matrices defining the state-trajectory associated with the dynamics (2). First, we recall that a weighted adjacency matrix $A$ associated with a graph $\overline{\mathcal{G}}=(\overline{\mathcal{V}}, \overline{\mathcal{E}})$ is a matrix with non-negative entries satisfying $A_{i, j}>0 \Leftrightarrow(i, j) \in \overline{\mathcal{E}}$. The corresponding degree matrix $D$ is diagonal and $D_{i, i}=\sum_{j=1}^{n} A_{i, j}$. The weighted Laplacian matrix associated with $\overline{\mathcal{G}}$ is simply defined as $\bar{L}=D-A$. 
Proposition 2 Let $\overline{\mathcal{G}}$ be a directed graph with $n$ vertices containing a spanning tree with the root $\ell$. Let $A$ be a weighted adjacency matrix associated with $\overline{\mathcal{G}}$ and $D, \bar{L}$ the corresponding degree and weighted Laplacian matrices. Then $e^{-\bar{L} t}$ is a stochastic matrix for all $t \geq 0$ and

$$
\begin{aligned}
& \forall \delta>0 \exists \bar{\alpha}>0, \delta_{M}>\delta \text { such that } \\
& \left\{\begin{array}{l}
\left(e^{-\bar{L} t}\right)_{i, \ell}>\bar{\alpha}, \\
\left(e^{-\bar{L} t}\right)_{i, i}>\bar{\alpha}
\end{array}, \forall i \in\{1, \ldots, n\}\right.
\end{aligned}
$$

for all $t \in\left[\delta, \delta_{M}\right]$.

Remark 3 Proposition 2 shows that Assumption 3 (Dwell time) is the corresponding of Assumption 2 (Minimal influence) for the continuous dynamics defined by $L_{i}$.

Remark 4 For a given $\delta$, Proposition 2 states that for all $i \in\{1, \ldots, m\}$ it exists $\alpha_{i}$ such that $\left(e^{-L_{i} t}, \alpha_{i}\right)$ satisfies (8).Nevertheless, since we have a finite number of matrices $L_{i}$, we can consider a unique value $\alpha=\min \alpha_{i}$ satisfying Assumption 2 on one hand and $\left(e^{-L_{i} t}, \alpha\right)$ satisfies (8).

\subsection{Convergence analysis}

This part contains the main results of the paper concerning fully decentralized reset rules. In this section, Assumption 3 (Dwell time) is required but overlapping of the intervals $\left(t_{\phi_{i}(k)}, t_{\phi_{i}(k+1)}\right)$ and $\left(t_{\phi_{j}(h)}, t_{\phi_{j}(h+1)}\right)$ are allowed. This means, $t_{k+1}-t_{k}$ can be arbitrarily small and the existing results in the literature are not applicable. Under such assumption we will show that all agents eventually converge toward the same consensus state at exponential speed (Theorem 7). Prior stating the main result we provide the necessary intermediate ingredients.

For all time $t \in \mathbb{R}_{+}$, we define the global diameter of the group as

$$
\Delta(t)=\bar{x}(t)-\underline{x}(t)
$$

with

$$
\bar{x}(t)=\max _{i \in\{1, \ldots, n\}} x_{i}(t) \text { and } \underline{x}(t)=\min _{i \in\{1, \ldots, n\}} x_{i}(t) .
$$

Our goal in the sequel is to show that $\Delta(t)$ approaches 0 when $t$ increases. This requires some intermediate results presented as Lemmas in the sequel. All of them are written in terms of minimum $\underline{x}(t)$ but they can be easily transformed in terms of maximum $\bar{x}(t)$.

\section{Summary}

- In Lemma 3 we prove that: if an agent resets its state by taking into account a state bigger than the minimum, then its state after reset will be bigger than the minimum. So, one ensures that, after a reset at time $t_{\phi_{i}(k)}$, the state of one root of cluster $\mathcal{C}_{i}$ will not be arbitrarily close to the minimum $\underline{x}(t)$.

- In Lemma 4 we prove that during the continuous dynamics the root of a cluster will pull all the states of the corresponding cluster far from the minimum value. Before the next reset concerning this cluster, none of its state will be arbitrarily close to the minimum $\underline{x}(t)$. 
- In Lemma 5 we combine Lemma 3 and Lemma 4 in order to show that along a path of clusters the states cannot be arbitrarily close to the minimum $\underline{x}(t)$.

- Finally, in Theorem 7 we use the Lemmata to prove the geometric decreasing of the diameter $\Delta(t)$.

Lemma 3 (Reset) Let $i \in\{1, \ldots, m\}$ and $t \geq 0$ fixed. Let $t_{\phi_{i}(k)}>t$ be some reset instant. Assume that there are some $\ell \in \mathcal{V}$, some bound $X \in \mathbb{R}_{+}$, some $j \in \mathcal{C}_{i}$ and some bound $\alpha \in[0,1]$ such that

$$
x_{\ell}\left(t_{\phi_{i}(k)}^{-}\right)-\underline{x}(t) \geq X \text { and } P_{j, \ell}\left(t_{\phi_{i}(k)}\right) \geq \alpha .
$$

Then, we have

$$
x_{j}\left(t_{\phi_{i}(k)}\right)-\underline{x}(t) \geq \alpha X .
$$

Lemma 4 (Continuous dynamics) Let $i \in\{1, \ldots, m\}$ and $t \geq 0$ fixed. Let $k \in \mathbb{N}$ such that $t_{\phi_{i}(k)}>t$ and introduce the matrix $R=e^{-L_{i}\left(t_{\phi_{i}(k+1)}-t_{\phi_{i}(k)}\right)}$. Assume that for the root $\ell$ of one spanning tree of the cluster $\mathcal{C}_{i}$ there exist some bounds $Y \in \mathbb{R}_{+}$ and $\alpha \in[0,1]$ such that

$$
x_{\ell}\left(t_{\phi_{i}(k)}\right)-\underline{x}(t) \geq Y \text { and } \forall j \in \mathcal{C}_{i}, R_{j, \ell} \geq \alpha .
$$

Then, we have

$$
\min _{j \in \mathcal{C}_{i}} x_{j}\left(t_{\phi_{i}(k+1)}^{-}\right)-\underline{x}(t) \geq \alpha Y .
$$

Before giving the next result let us introduce some notation that will simplify the presentation. We say $\left(\mathcal{C}_{1}, \ldots, \mathcal{C}_{q}\right)$ is a path of clusters if for all $k \in\{1, \ldots, q-1\}$ there exists at least a directed link $\left(i_{k}, j_{k}\right)$ such that $i_{k} \in \mathcal{C}_{k}$ and $j_{k} \in \mathcal{C}_{k+1}$. Let us consider a generic path of clusters $\left(\mathcal{C}_{1}, \ldots, \mathcal{C}_{q}\right)$ such that for every $k \in\{1, \ldots, q-1\}$, there is $\ell$ in cluster $\mathcal{C}_{k}$ and $r$ a root of $\mathcal{C}_{k+1}$ for which $(\ell, r) \in \mathcal{E}$. Let $t \geq 0$ be fixed. We define a sequence of integers $\left(k_{1}, \ldots, k_{q}\right)$ such that:

- $t_{\phi_{1}\left(k_{1}\right)}$ is the first reset instant after $t$ of a root of a spanning tree of cluster $\mathcal{C}_{1}$, if a root reseting its state exists in $\mathcal{C}_{1}$. This may not be the case since $\mathcal{C}_{1}$ may not be influenced by the other clusters (according to Assumption 1), then $t_{\phi_{1}\left(k_{1}\right)}=t$ and $t_{\phi_{1}\left(k_{1}+1\right)}=t+\delta$.

- For all $h \in\{2, \ldots, q\}$, we define $t_{\phi_{h}\left(k_{h}+1\right)}$ the first instant after $t_{\phi_{h}\left(k_{h}\right)}$ when a root of a spanning tree of $\mathcal{C}_{h}$ resets its state.

- For all $h \in\{1, \ldots, q-1\}$ we define $t_{\phi_{h+1}\left(k_{h+1}\right)}$ as the first reset instant of a root of a spanning tree of cluster $\mathcal{C}_{h+1}$ after time $t_{\phi_{h}\left(k_{h}+1\right)}$.

Thus,

$$
\begin{gathered}
t<t_{\phi_{1}\left(k_{1}\right)}<t_{\phi_{1}\left(k_{1}+1\right)}<t_{\phi_{2}\left(k_{2}\right)}<t_{\phi_{2}\left(k_{2}+1\right)}< \\
\ldots<t_{\phi_{q}\left(k_{q}\right)}<t_{\phi_{q}\left(k_{q}+1\right)} .
\end{gathered}
$$

It is noteworthy that

$$
t_{\phi_{h+1}\left(k_{h+1}\right)}-t_{\phi_{h}\left(k_{h}+1\right)} \leq t_{\phi_{h+1}\left(k_{h+1}\right)}-t_{\phi_{h+1}\left(k_{h+1}-1\right)} \leq \delta_{\max } .
$$

Let also introduce $\mu=i p\left(\frac{\delta_{\max }}{\delta}\right)$ where $i p(y)$ denotes the biggest integer smaller than $y$. 
Remark 5 We have at most $\mu$ resets of a root of cluster $\mathcal{C}_{h}$ between $t_{\phi_{h}\left(k_{h}+1\right)}$ and $t_{\phi_{h+1}\left(k_{h+1}\right)}$.

In the sequel, iteratively applying Lemmas 3 and 4 we show that $\Delta\left(t_{\phi_{q}\left(k_{q}+1\right)}\right)$ geometrically decreases. For the next result we assume that a root $r$ of one tree in $\mathcal{C}_{1}$ satisfies

$$
x_{r}\left(t_{\phi_{1}\left(k_{1}\right)}\right)-\underline{x}\left(t_{\phi_{1}\left(k_{1}\right)}^{-}\right) \geq \Delta\left(t_{\phi_{1}\left(k_{1}\right)}\right) / 2 .
$$

If it is not the case, we instead consider the system where all the states have been reversed : $x_{i}:=-x_{i}$ and apply the same reasoning. In other words we relate the reasoning to the maximum instead of the minimum. In the sequel, we use $\underline{x}_{\mathcal{C}_{h}}(t) \triangleq$ $\min _{i \in \mathcal{C}_{h}} x_{i}(t)$.

Lemma 5 (Path of clusters) For all $h \in\{1, \ldots, q\}$, we have

$$
\underline{x}_{\mathcal{C}_{h}}\left(t_{\phi_{h}\left(k_{h}+1\right)}^{-}\right)-\underline{x}\left(t_{\phi_{1}\left(k_{1}\right)}^{-}\right) \geq \frac{\alpha^{(\mu+2)(h-1)+1} \Delta\left(t_{\phi_{1}\left(k_{1}\right)}\right)}{2} .
$$

Due to Assumption 1 we can reorder the clusters such that there exists a tree satisfying the following:

- its root is contained in $\mathcal{C}_{1}$;

- for all $i \geq 2$ there exist $j<i, \ell_{j} \in \mathcal{C}_{j}$ and $r_{i}$ a root of $\mathcal{C}_{i}$ such that $\left(\ell_{j}, r_{i}\right) \in \mathcal{E}$.

Proposition 6 There exists some non-negative integer $k \leq 2 m-1$ such that for all $t \in \mathbb{R}_{+}$,

$$
\underline{x}\left(k \delta_{\max }+t_{\phi_{1}\left(k_{1}\right)}^{-}\right)-\underline{x}\left(t_{\phi_{1}\left(k_{1}\right)}^{-}\right) \geq \frac{\alpha^{(\mu+2)(m-1)+1} \Delta\left(t_{\phi_{1}\left(k_{1}\right)}\right)}{2} .
$$

Once Proposition 6 is given, the exponential decay of the network diameter comes easily.

Theorem 7 There exists some positive decay rate $\beta \in[0,1)$ such that for all $t \in \mathbb{R}_{+}$,

$$
\Delta\left(2 m \delta_{\max }+t\right) \leq \beta \Delta(t)
$$

\subsection{Numerical examples}

In the following we consider a network of five agents grouped in two clusters. The network structure satisfies Assumption 1 and is described by the following Laplacian matrix:

$$
L=\left(\begin{array}{ccccc}
3 & 0 & -3 & 0 & 0 \\
-1 & 1 & 0 & 0 & 0 \\
-2 & 0 & 2 & 0 & 0 \\
0 & 0 & 0 & 0 & 0 \\
0 & 0 & 0 & -1 & 1
\end{array}\right)
$$

which has a block diagonal structure corresponding to the two clusters. Each cluster contains only one node able to interact with agents outside its own cluster (node 1 in the first cluster and node 4 in the second cluster). The weights of the inter-clusters interactions are chosen as follows 


$$
P=\left(\begin{array}{ccccc}
0.7 & 0 & 0 & 0.3 & 0 \\
0 & 1 & 0 & 0 & 0 \\
0 & 0 & 1 & 0 & 0 \\
0.25 & 0 & 0 & 0.75 & 0 \\
0 & 0 & 0 & 0 & 1
\end{array}\right),
$$

such that Assumption 2 holds. We point out that at reset times $t_{k}$ either only one or both nodes 1 and 4 reset their state. Therefore, the matrices $P\left(t_{k}\right)$ are either equal $P$ or obtained by replacing the first or forth line of $P$ by $(1,0,0,0,0)$ or $(0,0,0,1,0)$, respectively. Assumptions 3 and 4 are guaranteed by the choice of $\delta=4$ and $\delta_{\max }=8$.

In Figure 1 we firstly emphasize the agreement of all five agents. A zoom-in allows to point out that each impulsive agent resets its state in its own rhythm and it may happen that one of them resets twice between the reset times of the other.

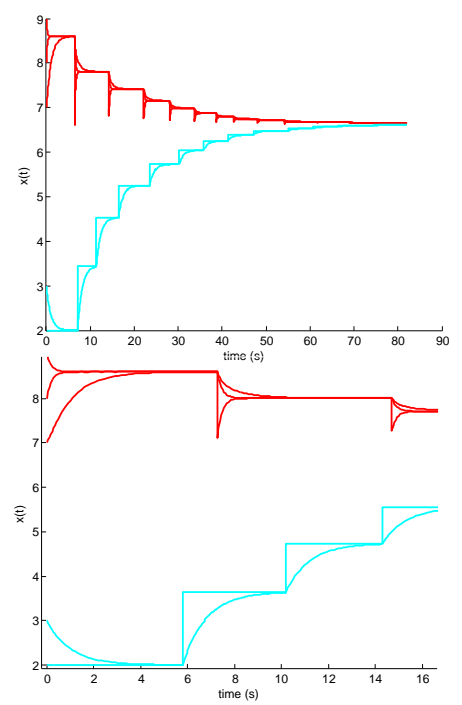

Figure 1: Up: Consensus of the five agents grouped in 2 clusters. Down: Zoom in pointing out that the resets are not synchronized.

\subsection{Conclusions}

In this paper we have studied the consensus in heterogeneous networks containing both linear and linear impulsive dynamics. Under appropriate assumptions we have proven that all subsystems agree and we have upper-bounded the convergence speed. It is noteworthy that, since the reset instants are not synchronized, we cannot apply the existing results concerning the consensus of linear discrete dynamics in networks with dynamically changing interaction topologies. A small size network has been used to numerically illustrate the state trajectory of the network. 


\section{Bibliography}

[1] L. Xiao and S. Boyd, "Fast linear iterations for distributed averaging," System and Control Letters, vol. 53, pp. 65-78, 2004.

[2] A. Olshevsky and J. Tsitsiklis, "Convergence speed in distributed consensus and averaging," SIAM Journal of Control and Optimization, vol. 48, no. 1, pp. 33-55, 2009.

[3] A. Jadbabaie, J. Lin, and A. S. Morse, "Coordination of groups of mobile autonomous agents using nearest neighbor rules," IEEE Trans. on Automatic Control, vol. 48, no. 6, pp. 988-1001, 2003.

[4] R. Olfati-Saber and R. Murray, "Consensus problems in networks of agents with switching topology and time-delays," IEEE Transactions on Automatic Control, vol. 49, pp. 1520-1533, 2004.

[5] W. Ren and R. W. Beard, "Consensus seeking in multiagent systems under dynamically changing interaction topologies," IEEE Trans. on Automatic Control, vol. 50, no. 5, pp. 655-661, 2005.

[6] S. Martin and A. Girard, "Continuous-time consensus under persistent connectivity and slow divergence of reciprocal interaction weights." SIAM Journal on Control and Optimization, vol. 51, no. 3, pp. 2568-2584, 2013.

[7] J. M. Hendrickx and J. N. Tsitsiklis, "Convergence of type-symmetric and cutbalanced consensus seeking systems." IEEE Transactions on Automatic Control, vol. 58, no. 1, pp. 214-218, 2013.

[8] J. Mei, W. Ren, and G. Ma, "Distributed coordinated tracking with a dynamic leader for multiple Euler-Lagrange systems," IEEE Transactions on Automatic Control, vol. 56, no. 6, pp. 1415-1421, 2011.

[9] H. Su, G. Chen, X. Wang, and Z. Lin, "Adaptive second-order consensus of networked mobile agents with nonlinear dynamics," Automatica, vol. 47, no. 2, pp. 368-375, 2011.

[10] L. Buşoniu and I.-C. Morărescu, "Consensus for black-box nonlinear agents using optimistic optimization,” Automatica, vol. 50, no. 4, pp. 1201-1208, 2014.

[11] S. Martin and A. Girard, "Sufficent conditions for flocking via graph robustness analysis," in Proc. of the 49th IEEE Conference on Decision and Control, 2010, pp. 6293-6298. 
[12] M. Zavlanos and G. Pappas, "Distributed connectivity control of mobile networks," IEEE Transactions on Robotics, vol. 24, no. 6, pp. 1416-1428, 2008.

[13] M. Fiacchini and I.-C. Morărescu, "Convex conditions on decentralized control for graph topology preservation," IEEE Transactions on Automatic Control, vol. 59, no. 6, pp. 1640-1645, DOI.10.1109/TAC.2013.22927192014.

[14] I.-C. Morărescu and A. Girard, "Opinion dynamics with decaying confidence: Application to community detection in graphs," IEEE Trans. on Automatic Control, vol. 56, no. 8, pp. $1862-1873,2011$.

[15] B. Touri and A. Nedic, "On approximations and ergodicity classes in random chains," IEEE Transactions on Automatic Control, vol. 57, no. 11, pp. 2718 2730, 2012.

[16] O. Beker, C. Hollot, and Y. Chait, "Plant with an integrator: an example of reset control overcoming limitations of linear feedback," IEEE Transactions on Automatic Control, vol. 46, no. 11, pp. 1797-1799, 2001.

[17] D.Nešić, L. Zaccarian, and A. R. Teel, "Stability properties of reset systems," Automatica, vol. 44, pp. 2019-2026, 2008.

[18] A. Banos, J. Carrasco, and A. Barreiro, "Reset times-dependent stability of reset control systems," IEEE Transactions on Automatic Control, vol. 56, no. 1, pp. 217-223, 2011.

[19] M. C. Bragagnolo, I.-C. Morărescu, J. Daafouz, and P. Riedinger, "Lmi sufficient conditions for the consensus of linear agents with nearly-periodic resets," in American Control Conference, 2014.

[20] V. D. Blondel, J. M. Hendrickx, A. Olshevsky, and J. Tsitsiklis, "Convergence in multiagent coordination, consensus, and flocking," in Proc. IEEE Conf. on Decision and Control, 2005, pp. 2996-3000. 